\title{
Prognostic Implication of Positive Area on Bone Scans (\%PAB- Quantitative Parameter) in Bones of Patients with metastatic Prostate cancer
}

\author{
Nayab Mustansar* \\ Women Medical College, Abbottabad, Pakistan
}

*Corresponding author: Nayab Mustansar, Assistant Professor, Consultant Nuclear

Physician, Women Medical College, Abbottabad, Pakistan.

Received Date: June 24, 2019

Published Date: June 28, 2019

\section{Introduction}

Prostate Cancer is one of the common cancers in the world. It could primarily disseminate to the bone and can lead to death. In order to address its life-threatening distant metastasis, it is important to diagnose it earlier for timely treatment. Bone metastasis is usually diagnosed deploying bone scan imaging. However, interpretation of the bone scans is a tedious procedure for the physicians and often leads to misinterpretation either as overestimation or underestimation of the metastasis. To minimize the risk of misinterpretation, one of the accurate methods is quantitative analysis of the bone scans in order to ascertain, whether a metastatic lesion is present or not. There are several methods to-date which can be used to analyze the extent of such lesions. The aim of this study is to use \% PAB (Positive Area on Bone Scan-quantitative parameter) on a baseline bone scan.

Moreover, an improved methodology is introduced by comparing the results with PSA levels. 141 patients with histopathologically proved prostate cancer were chosen to implement the $\%$ PAB on individual baseline bone scans. After which, for the calculation of risk of progression or regression of disease and survival rate, 40 patients were chosen from the same dataset. A serial follows up scan was performed to calculate 2-years survival rate. The dataset was again analyzed using the same quantitative parameter and the cut off were calculated as \% PAB: 0.5 .

It was found out that the $\%$ PAB method is a good prognostic indicator in baseline scans. Moreover, the prostate cancer patients with the cut off \% BSI > 0.5 showed increase risk of disease progression and less survival.

\section{Acknowledgement}

I want to pay my humble gratitude to the constant support of my husband Capt Dr Rizwan Rafi and my little angel daughter "Hadees Rafi" who is my pleasant mood modulator and of Course the never ending prayers of my parents in every pace of my Life. Without all of them I have never been able to succeed.

\section{Conflict of Interest}

No conflict of interest. 